\title{
A potential application of a piezoelectric atomiser for ophthalmic drug delivery
}

doi:10.1080/11762320701403906

\author{
Wei Xia ${ }^{1}$, Hung-Yao Hsu' ${ }^{1}$ Lingxue Kong ${ }^{1}$, Allan Evans ${ }^{2}$ and \\ Ryan Lee ${ }^{3}$ \\ ${ }^{I}$ Centre for Material and Fibre Innovation, Deakin University, Australia \\ ${ }^{2}$ Division of Health Sciences, University of South Australia, Adelaide, Australia \\ ${ }^{3}$ Sunnytec Electronics Co., Ltd., Taiman
}

\begin{abstract}
A liquid atomiser composed of a piezoelectric transducer and a metal plate with numerous micro-apertures is studied to identify the most influential factors on its atomising performance. The Taguchi method is employed in the experiment design and analysis of the study on how each factor acts in the atomising process. An optimal condition is determined for producing a stream of droplets. The study shows that the droplet size and the spraying velocity are suitable for ophthalmic drug delivery application, with an even distribution of the drug over most of the eyeball surface area due to the controllable cross-sectional area of the droplet stream. This greatly improves the treatment effectiveness and efficiency of eye therapy. Finally, a structure of the ophthalmic drug delivery system is proposed.
\end{abstract}

Keywords: Piezoelectric atomiser, atomisation process, ophthalmic drug delivery, Taguchi method.

\section{INTRODUCTION}

Conventional eyedroppers have been used widely as the primary method for ocular drug delivery without significant change in the past few decades. Eyedroppers normally consist of three parts: a plastic or glass bottle to contain the ophthalmic solution, a dropper to dispense the solution, and a closure cap. However, considerable disadvantages and limitations of the eyedroppers have been pointed out by researchers, which are summarized as follows:

First, the application of eyedropper is problematic due to the high variability of the eye drop volume. The drop sizes of many commercially available topical ocular medications are reported to be from 25 up to $70 \mu \mathrm{L}$ (Shell 1982; Lederer and Harold 1986; Urtti and Salminen 1993). The variation of drop size is caused by three main factors: the dropper tip design, the physicochemical properties of the solution, and the patient's manner of handling the dropper bottle, such as the dispensing angle and rate (van Santvliet 1999; Sklubalova and Zatloukal 2005; German et al. 1997).

Corresponding Author:

Wei Xia

Centre for Advanced Manufacturing Research

University of South Australia

Adelaide, Australia

Tel: 61-8-83025561; Fax: 61-8-83025292

Email: wei.xia@postgrads.unisa.edu.au
Second, a major portion of each eye drop administered is blinked out and drained into the nasolacrimal duct system. This results in poor bioavailability of the drug and unwanted systemic side effects (Lee et al. 1999). Normally, the maximum volume an upright non-blinking eye is able to accommodate is estimated at $30 \mu \mathrm{L}$ (Yaniv and Hadar 1994; Mishima et al. 1966). Because of protective mechanisms, the eye will react intuitively to the suddenly increased volume from the stillation of eye drops. The solution is then diminished rapidly by reflex blinking, tearing, and increased rate of drainage. Over $90 \%$ of the administered eye drop is cleared within 2 minutes. Only $1 \%-10 \%$ of the administered dose penetrates to reach the inner eye. The bioavailability is typically less than $5 \%$ (Davies 2000; Geroski and Edelhauser 2000; Maurice and Mishima 1984). On the other hand, the drug solution draining into the nasolacrimal duct can be absorbed systematically through the nasopharyngeal and gastrointestinal mucosa. Several major systemic side effects have been reported, such as $\beta$-blockers asthma, reduced cardiovascular response to exercise (Diamond 1997; Diggory and Franks 1996; Jarvinen et al. 1995; O'Donoghue 1995; O'Sullivan et al. 2001) and respiratory arrest, especially for children who have smaller body sizes and lower systemic drug metabolizing capacities (van Santvliet and Ludwig 2004). From bioavailability, toxicological, and economic points of view, smaller drop volumes of $5-15 \mu \mathrm{L}$ should be instilled (Shell 1984; Urtti and Salminen 1993; Fiscella 1998). 
Third, the patient has to incline the face almost $90^{\circ}$ to ensure the eye drops are administered into the eyes under gravity. This is physically difficult for many patients, especially the elderly, which can lead to poor compliance (Winfield et al. 1990). Moreover, the impact of drug drops leads to discomfort to the patient's eyes.

Based on the above recognition, a vast amount of researches have been carried out on the development of ocular drug delivery systems in the past two decades. The common alternate option to eye drops is ophthalmic inserts. The inserts are solid devices intended to be placed in the conjunctival sac and to deliver the drug at a comparatively slow rate. Several advantages have been reported, such as prolonged drug activity and a higher bioavailability, accurate dosing, and possible reduction of systemic absorption. However, they have not gained acceptance owing to the reluctance of the psychologists and patients to abandon the traditional liquid medications, higher costs, and occasional therapeutic failure (Saettone 2002). Furthermore, it is difficult for patients to place and remove the inserts, and it can also cause discomfort and short-term blurred vision (Himmelstaein and Baustian 1997).

This research is aimed to develop an ophthalmic drug delivery device that can enhance the drug bioavailability, diminish systemic side effects and improve patient compliance. A novel system with a piezoelectric atomiser is proposed in this work. The targets of the drug delivery system are as follows:

- To apply with small and precise dosage (5-15 $\mu \mathrm{L})$ so that the drug solution will not be blinked out or drained into the nasolacrimal duct. This will minimize the systemic side effects and the waste of drug.

- To apply in the form of micro-droplets that is gentle to human eyes.

- To apply with droplets mist in elliptical shape that can fit the shape of the eye and be able to cover most of the eyeball surface area to achieve more efficient therapeutic contact.

- Easy to use.

\section{PIEZOELECTRIC ATOMIZER}

Piezoelectric atomiser has been studied for application in drug delivery, such as pulmonary drug delivery in diabetes therapy (Kong et al. 2002). The atomiser used is composed of a piezoelectric disk, a membrane of glass wafer and a silicon wafer with chambers and slots. Under the pressure of the piezoelectric disk glued on the glass membrane, drug liquid supplied to the chambers between the glass membrane and the silicon wafer is squeezed out from the tiny slots as a filament and breaks up into droplets due to Rayleigh instability. The drug droplets are then inhaled by patients. However, this device is not suitable for ophthalmic drug delivery because the directivity of the droplets broken up from the filament is not good and uncontrollable. Although a piezo atomiser that can be used for ophthalmic drug delivery is proposed in a patent (Davison

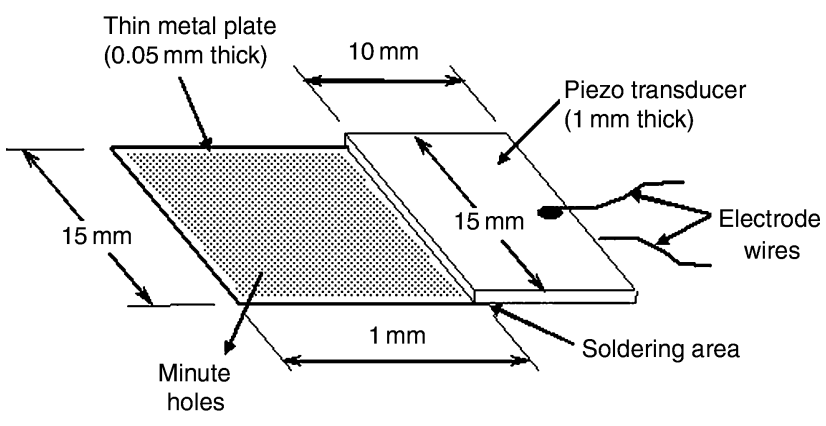

Figure 1 Schematics of the piezoelectric atomiser.

et al. 2000), the structure of the device is too complicated. The key elements of the device are a perforated membrane, an annular piezoelectric transducer, a liquid supply system, and an extension duct with air impeller. The membrane bonded on the bottom of the transducer vibrates when the transducer is excited. Drug supplied onto the upper surface of the membrane goes through the tiny apertures and becomes fine droplets. The drug droplets then have to be blown away by air impeller along the duct from one end to an eye at the other. This airflow can cause discomfort to human eyes.

The piezoelectric atomiser used in this work is composed of a transducer and a thin metal plate (Fig 1). The transducer is made of piezoelectric ceramic $15 \mathrm{~mm}$ long $\times$ $10 \mathrm{~mm}$ wide $\times 1 \mathrm{~mm}$ thick. Both top and bottom sides are coated with a silver film and an electrode wire is soldered on each side. The thin metal plate $(15 \mathrm{~mm}$ long $\times 15 \mathrm{~mm}$ wide $\times 0.05 \mathrm{~mm}$ thick) is made of $\mathrm{Fe}-\mathrm{Ni}$ alloy and coated with a gold film. There are numerous minute holes in the metal plate, which are fabricated in a matrix. The holes are conical, with the smaller end $16 \mu \mathrm{m}$ in diameter and the bigger end $50 \mu \mathrm{m}$. The central distance of two adjacent holes is $150 \mu \mathrm{m}$. The metal plate is soldered underneath the piezo transducer with the smaller end of the holes on the top side. The soldering area is $15 \mathrm{~mm} \times 2 \mathrm{~mm}$.

Toda and Ishll (1995) have studied a similar atomiser, whose focus is mainly on the construction of a selfoscillation driving circuit and the influence of the plate length on the atomiser's resonant frequency. This work will investigate the operating characteristics of the piezo atomiser and the influential factors, and further evaluate the feasibility of the atomiser for an effective ophthalmic drug delivery application.

The operating mechanism of the atomiser is shown in Figure 2. The metal plate is placed on a saturated sponge. Liquid is supplied to the underside of the metal plate. The transducer is driven by an ultrasonic wave signal. Liquid atomisation occurs under the action of surface acoustic waves excited by the piezo transducer (Kurosawa et al. 1995). At an appropriate resonant frequency and voltage, the transducer is excited to oscillate at a high frequency. The oscillation wave is then propagated to the metal plate attached. Liquid in the cavities formed by the conical holes of the metal plate is pressed by the pumping force and flow 


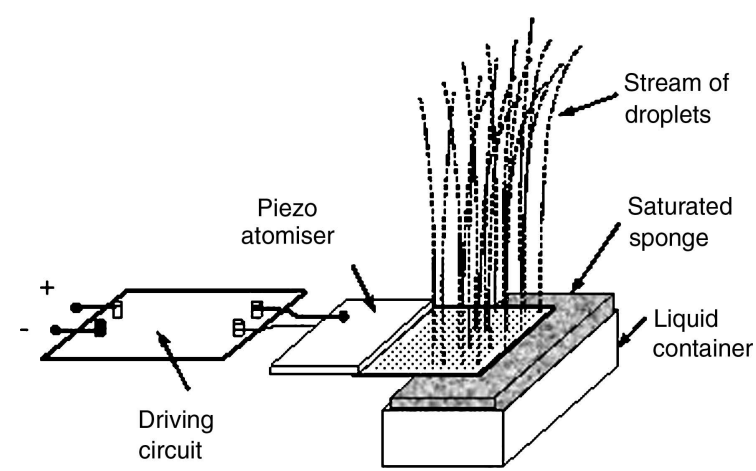

Figure 2 Operating mechanism of atomiser.

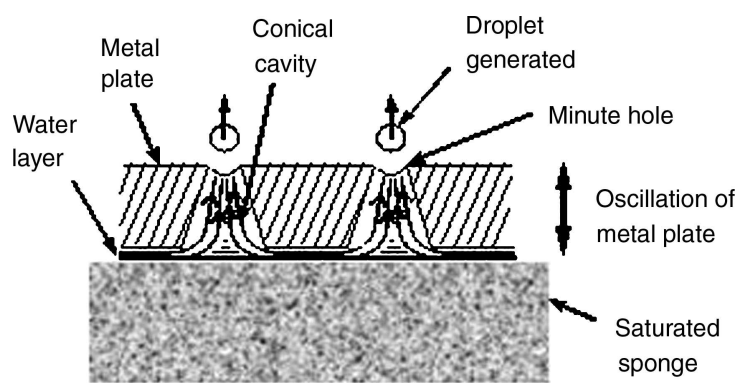

Figure 3 Mechanism of droplets formation.

through the minute holes, forming a stream of droplets (Fig 3). This process repeats continuously until power is removed or water supply is starved.

\section{EXPERIMENTAL STUDY ON THE CHARACTERISTICS OF THE ATOMIZER}

Seven influential factors on the atomisation process are identified for a detailed study. They are described as resonant frequency (RF), driving voltage (Volt), fixing method of the transducer $(\mathrm{FM})$, operating orientation (Ot), contact angle between the metal plate and the sponge (CA), gap between the metal plate and the sponge (Gp), collection distance between the metal plate and the droplets collection paper $(\mathrm{Dp})$.

\section{- Resonant frequency (RF)}

Piezo transducer is normally excited by sine wave signal at resonant frequency, where it oscillates with higher amplitude. Resonant frequencies of this atomiser are identified as $109,162,166,171,172,177$, and $184 \mathrm{kHz}$. Preliminary tests conducted on the atomiser by setting the output voltage of the signal generator at 28 volts peak-to-peak $(\mathrm{Vp}-\mathrm{p})$ and tuning the frequency of the sine wave signal around the abovementioned resonant frequencies. It reveals that the stream of droplets can only be generated at 171 and $172 \mathrm{kHz}$. At other resonant frequencies, the atomiser only generates a mist layer $5 \mathrm{~mm}$ thick above the metal plate, which is not suitable for drug delivery. Resonant frequencies of 171 and $172 \mathrm{kHz}$ are to be used for the study.

- Driving voltage (Volt)

Voltage determines the oscillation amplitude of the trans-

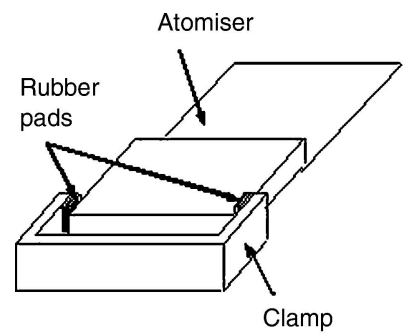

(a)

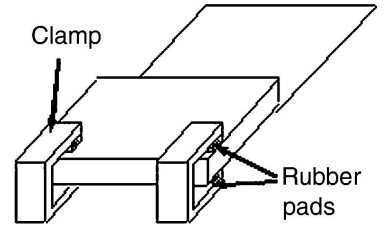

(b)

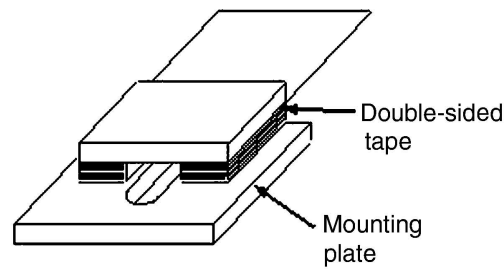

(c)

Figure 4 Fixing methods of the atomiser on (a) side; (b) top-bottom; (c) sticking.

ducer. At threshold voltage, water underneath the metal plate passes through the minute holes due to the pumping force generated by the oscillation of the plate. Droplets are formed on the top surface of the metal plate but the energy supplied is not high enough for the droplets to overcome the water surface tension to escape from the metal plate (Kurosawa et al. 1995). When higher voltage is supplied, more droplets are produced and escape from the plate with a certain velocity, forming a flowing beam. The threshold voltage at 171 and $172 \mathrm{kHz}$ was tested to be $12-14 \mathrm{Vp}-\mathrm{p}$; 16 and $24 \mathrm{Vp}-\mathrm{p}$ are used for the experiment.

- Fixing methods (FM)

There are three fixing methods as shown in Figure 4: (a) clamping on the sides; (b) clamping on the top-bottom surfaces; and (c) sticking on a plate with a double-sided tape. Rubber pads $2 \mathrm{~mm}$ thick are used between the clamp and the transducer for type (a) and (b). The dimension of the double-sided tape in (c) is $15 \mathrm{~mm}$ long $\times 10 \mathrm{~mm}$ wide $\times 1.5 \mathrm{~mm}$ thick, with a cut-off area of $5 \mathrm{~mm} \times 5 \mathrm{~mm}$ to allow for the electrode wire soldered on the transducer.

- Operating orientation (Ot)

The atomiser can be either placed on top or at a vertical side of the saturated sponge.

- Contact angle (CA)

It is the angle between the sponge surface and bottom surface of the metal plate; $0^{\circ}$ and $5^{\circ}$ are studied.

- Gap (Gp)

It is the gap between the metal plate and the sponge; 0 and $0.5 \mathrm{~mm}$ are compared.

- Collection distance (Dp)

The distance between the metal plate surface and the collection paper is used for studying the changes in shape and size of the cross section of the droplet stream and the amount of droplets collected. Distances of 10 and $40 \mathrm{~mm}$ are used for the experiment.

The above seven factors and their levels are shown in Table 1. 
Table 1 Influential factors and levels

\begin{tabular}{llllllll}
\hline & \multicolumn{7}{c}{ Factors } \\
\cline { 2 - 7 } Levels & FM & CA $\left({ }^{\circ}\right)$ & Ot & RF $(\mathrm{kHz})$ & Volt $(\mathrm{Vp}-\mathrm{p})$ & Dp $(\mathrm{mm})$ & $\mathrm{Gp}(\mathrm{mm})$ \\
\hline 1 & Side & 0 & Horizontal & 171 & 16 & 1 & 0 \\
2 & Top-bottom & 5 & Vertical & 172 & 24 & 40 & 0.5 \\
3 & Stick & $\ldots$ & $\ldots$ & $\cdots$ & $\cdots$ & $\ldots$ & $\cdots$ \\
\hline
\end{tabular}

Table 2 Twelve trial testing conditions

\begin{tabular}{rlllllll}
\hline & \multicolumn{7}{c}{ Factors } \\
\cline { 2 - 7 } Trial no. & FM & CA $\left(^{\circ}\right)$ & Ot & RF $(\mathrm{kHz})$ & Vol (Vp-p) & Dp $(\mathrm{mm})$ & $\mathrm{Gp}(\mathrm{mm})$ \\
\hline 1 & Side & 0 & Horizontal & 171 & 16 & 10 & 0 \\
2 & Side & 0 & Horizontal & 172 & 24 & 40 & 0.5 \\
3 & Side & 5 & Vertical & 171 & 16 & 40 & 0.5 \\
4 & Side & 5 & Vertical & 172 & 24 & 10 & 0 \\
5 & Top-bottom & 0 & Vertical & 171 & 24 & 10 & 0.5 \\
6 & Top-bottom & 0 & Vertical & 172 & 16 & 40 & 0 \\
7 & Top-bottom & 5 & Horizontal & 171 & 24 & 40 & 0 \\
8 & Top-bottom & 5 & Horizontal & 172 & 16 & 10 & 0.5 \\
9 & Stick & 0 & Horizontal & 171 & 24 & 40 & 0.5 \\
10 & Stick & 0 & Vertical & 172 & 16 & 10 & 0.5 \\
11 & Stick & 5 & Horizontal & 172 & 24 & 10 & 0 \\
12 & Stick & 5 & Vertical & 171 & 16 & 40 & 0 \\
\hline
\end{tabular}

In order to obtain the most information about the effects of these factors on the atomisation performance with the fewest trials, Taguchi method is utilized for the experiment design and analysis. Taguchi method is a statistical method that uses representative fractional factorial experiments (FFEs) to determine optimal parameters and fully investigate the variability, especially the sensitivity of the system specification to the causal factors of variability (Manton 1989). This method provides an efficient and cost-effective way for the identification of influential factors and optimization of parameters compared with a full-factorial design. In FFE designs, the orthogonal array uses only a portion of the total possible combinations to estimate the main factor effects and some of the interactions. Certain representative treatment conditions are chosen to maintain the orthogonal relationship among the various factors and interactions, for example, the L8 $\left(2^{7}\right)$ Array and the L9 $\left(3^{4}\right)$ Array. The former one, having eight trial conditions, is applicable for seven factors with two levels of setting. The latter, with nine trial conditions, is for four factors with three levels (Ross, 1988).

Among the seven factors, six have two levels, and only factor FM has three levels. Therefore, L8 Array is used and modified with four additional trial conditions to form a 12-trial testing plan (Table 2).

Instruments and materials used in the experiments are as follows:

1. Function generator: Model: Mini-Lab 603B (0-2 MHz)

2. Oscilloscope: TRIO CS-1.22 (20 MHz)
3. Frequency counter: Frequency/Period Counter (0-50 $\mathrm{MHz})$

4. Electric scale: Mettler H6 (106 g, $0.1 \mathrm{mg}$ accuracy)

5. Stop watch

6. Experimental rig (self-designed and made)

7. Sponge - highly absorbent

8. Collection paper and pad - highly absorbent

9. Clean water

In the experiment, water is used as the testing liquid. As this research is a preliminary feasibility study on the atomiser, it is impossible to finance a costly testing liquid, such as an eye drug. In effect, fluidic properties do not change the nature of the atomisation process but only affect its starting condition. A more precise experiment could be conducted with eye drug liquid in the future.

The experimental setup is shown in Figure 5. The sponge is placed in a container filled with water. The top surface is at $20 \mathrm{~mm}$ above the water level. The atomiser is held by the mounting rig. The metal plate is placed on the saturated sponge with contact area of $15 \mathrm{~mm} \times 5 \mathrm{~mm}$. The function generator is used to supply sine wave signals to drive the atomiser. The absorbent collection paper or pad is positioned at a preset distance above the atomiser.

Experiments are conducted three times under each trial condition shown in Table 2. The atomisation time for each operation is set as $10 \mathrm{~s}$. The collection paper is then weighed by an electric scale for measuring the mass of droplets collected. The collection pad is used to monitor the change of the cross-sectional area of the droplet stream by examining 


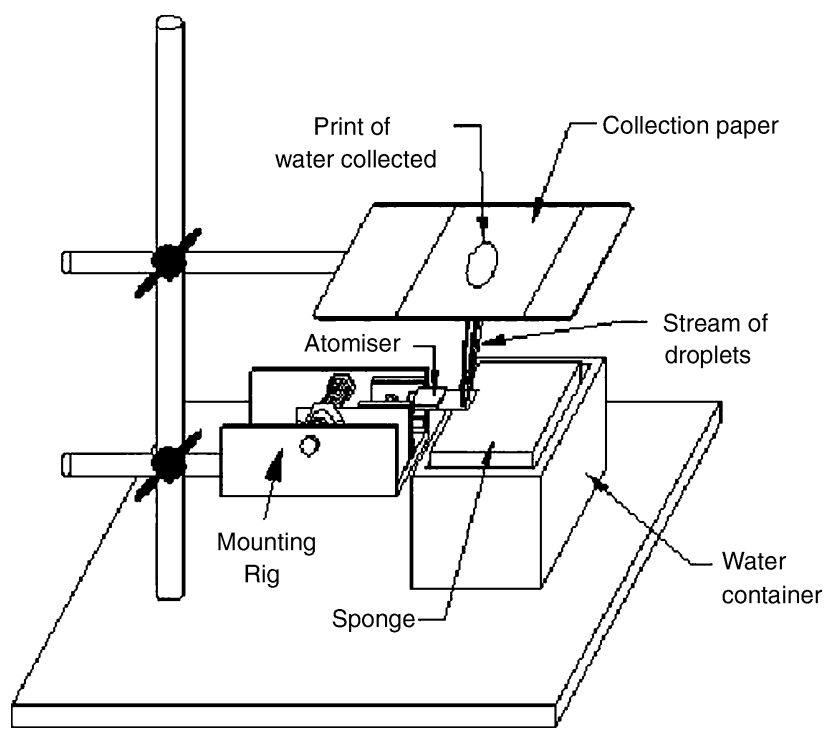

Figure 5 Experimental setup.

the water prints on the pad collected at preset positions above the atomiser.

\section{RESULTS AND DISCUSSION}

The mass of droplets collected in the experiment is weighed ranging from 1.08 to $2.92 \mathrm{~g}$ for the various trial conditions.

The experiment is analyzed based on the direct responses and the signal-to-noise $(\mathrm{S} / \mathrm{N})$ ratio. The direct responses, representing the performance characteristics, advise the experimenter how good the testing result is under a particular condition. In this experiment, the direct response is the mean value of the mass of the atomised water. The $\mathrm{S} / \mathrm{N}$ ratio is the ratio of the mean value (signal) to the standard deviation (noise). Taguchi method uses $\mathrm{S} / \mathrm{N}$ ratio to quantitatively analyse the variations of the performance indicator. In general, the $\mathrm{S} / \mathrm{N}$ ratio should be maximized to achieve a robust design. Three major $\mathrm{S} / \mathrm{N}$ ratios are applicable to a wide range of response variables, depending on particular analytical characteristics, which includes 'bigger is better' $\left(\mathrm{S} / \mathrm{N}_{\mathrm{B}}\right)$, 'nominal is better' $\left(\mathrm{S} / \mathrm{N}_{\mathrm{N}}\right)$ and 'smaller is better' $\left(\mathrm{S} / \mathrm{N}_{\mathrm{S}}\right)$ (Ross, 1988). For the analysis in this study, the first one is selected because the more droplets are generated the better. The equation is given by

$$
S / N_{B}=-10 \log _{10}\left(\frac{1}{n} \sum_{i=1}^{n} \frac{1}{y_{i}^{2}}\right)
$$

Where $\mathrm{n}$ is the number of trial repetitions under the same condition, $\mathrm{y}_{\mathrm{i}}$ is the result of each measurement.

The mean value of the droplets collected and $\mathrm{S} / \mathrm{N}$ ratio corresponding to the influential factors are graphed and shown in Figures 6 and 7, respectively.

The analytical diagrams reveal that voltage, fixing method, and operating orientation make distinctive influence on the atomisation process since larger slope of the line presents in the diagrams titled "Voltage", "Orientation", and "Fixing method" than other factors.

\section{Voltage}

Voltage determines the amplitude of the oscillation. Lower than the threshold voltage, the atomisation will not happen. In the Voltage diagram in Figure 6, the mean value of droplets collected under condition at $24 \mathrm{Vp}-\mathrm{p}(2.44 \mathrm{~g})$ is much more than that at $16 \mathrm{Vp}-\mathrm{p}(1.38 \mathrm{~g})$. A similar trend for $\mathrm{S} / \mathrm{N}$ ratio is presented in Figure 7. The $\mathrm{S} / \mathrm{N}$ ratio is bigger (better) at $16 \mathrm{Vp}-\mathrm{p}$ than that at V2. This indicates that the driving voltage determines the oscillation amplitude of the transducer. Both the atomisation rate and droplet velocity rise with the increase of driving voltage.

\section{Orientation}

According to the physical characteristics of the transducer, the orientation of the atomiser does not influence the atomisation performance. However, the diagrams for operating orientation show that the horizontally placed atomiser (mean value $2.18 \mathrm{~g}$ ) is more effective for atomisation than the vertically placed one $(1.64 \mathrm{~g})$. Although gravity affects the movement of the droplets while placed in vertical orientation, this effect is negligible. This will be discussed in
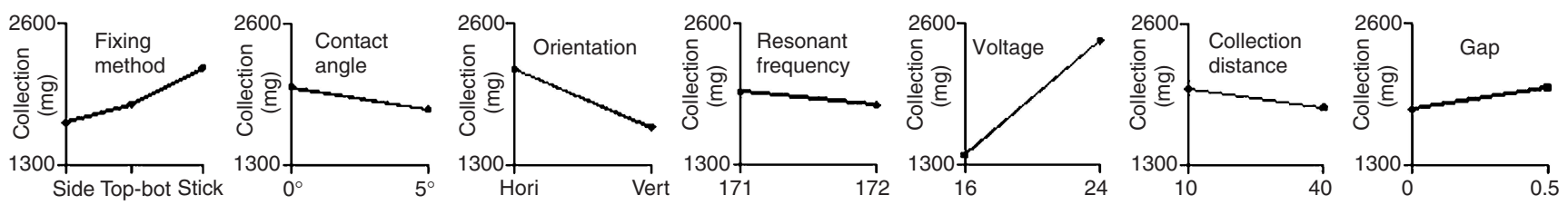

Figure 6 Mean value of droplets collected corresponding to the factors.
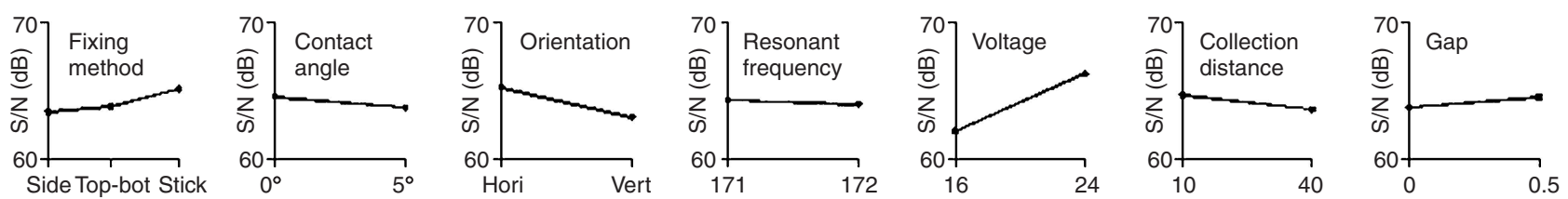

Figure $7 \mathrm{~S} / \mathrm{N}$ ratio corresponding to the factors. 
a later section. The difference shown in the experiment is due to the limitation of the sponge. When testing on the atomiser in vertical position, the atomiser is in contact with the side surface of the sponge above the water level of the container. The sufficiency of water supply to the atomiser and water distribution along the vertical direction in the sponge are largely determined by the capillary effects of the sponge and in turn influence the atomisation process. The available sponge used in the experiment is not an ideal one from this point of view. Further study is required to source a more efficient material that can absorb water evenly and that will improve the performance of the atomiser in this aspect.

\section{Fixing method}

The mean value of droplets collected is less with the transducer that is clamped on the sides $(1.69 \mathrm{~g})$ and top-bottom surfaces $(1.86 \mathrm{~g})$ than that with sticking method $(2.19 \mathrm{~g})$. Both analyses present the same trend. When the transducer being clamped on side or top-bottom, the holding pressure acting on the transducer constrains the oscillation in the "thickness" direction, which in turn weakens the atomisation effects. While with the sticking method, the bottom surface of the transducer is stuck on the mounting plate, leaving the top surface free from constraint. This provides more flexibility for the transducer to oscillate along the "thickness" direction. Therefore, "stick" is the best way among the three for fixing the atomiser.

\section{Resonant frequency}

As mentioned earlier, resonant frequency is also a decisive factor since atomisation is effective only at the major ones of the identified resonant frequencies, such as 171 and $172 \mathrm{kHz}$ for this transducer. The analyses shown in Figures 6 and 7 only indicate that the performance of the atomisation is quite similar at $171 \mathrm{kHz}(1.97 \mathrm{~g})$ and $172 \mathrm{kHz}$ $(1.85 \mathrm{~g})$, and the former is a little better. Actually, at other resonant frequencies, such as 109 and $184 \mathrm{kHz}$, the droplets are atomised and stay at the metal surface with nearly no spray.

Comparatively, the following three factors do not contribute as much to the atomisation process as the above ones.

\section{Gap between the metal plate and the sponge}

In this research, two gaps are tested, 0 and $0.5 \mathrm{~mm}$. The analyses (Figs 6 and 7) indicate that the atomisation is slightly stronger with the gap of $0.5 \mathrm{~mm}(2.01 \mathrm{~g})$ than that of $0 \mathrm{~mm}(1.81 \mathrm{~g})$. It is observed that the atomisation is sensitive to the size of the gap at lower voltages, such as $16 \mathrm{Vp}-\mathrm{p}$, but the sensitivity is diminished when higher voltage $(24 \mathrm{Vp}-\mathrm{p})$ is supplied.

When the metal plate contacts the sponge with no gap $(0 \mathrm{~mm})$, the metal plate is "sucked" by the sponge because of the surface tension of water. This constrains the os-
Table 3 The optimal operating condition

\begin{tabular}{lllllll}
\hline $\begin{array}{l}\text { RF } \\
(\mathrm{kHz})\end{array}$ & $\begin{array}{l}\text { Volt } \\
(\mathrm{Vp}-\mathrm{p})\end{array}$ & $\mathbf{F M}$ & $\mathbf{O t}$ & $\begin{array}{l}\text { CA } \\
\left({ }^{\circ}\right)\end{array}$ & $\begin{array}{l}\text { Dp } \\
(\mathrm{mm})\end{array}$ & $\begin{array}{l}\text { Gp } \\
(\mathrm{mm})\end{array}$ \\
\hline 171 & 24 & Stick & Horizontal & 0 & 10 & 0.5 \\
\hline
\end{tabular}

cillation of the metal plate and consequently weakens the atomisation effects.

When a proper gap exists between the metal plate and the sponge $(0.5 \mathrm{~mm})$, a thin layer of water is formed underneath the metal plate, which ensures water supply to the metal plate and the constraint from the sponge to the oscillation of the plate no longer exists. However, when a bigger gap exists between the plate and sponge $(>1 \mathrm{~mm})$, water cannot be transferred from the sponge to the metal plate and atomisation is then not possible.

\section{Contacting angle}

The experiment result indicates that the atomiser performs a little better when the metal plate is placed parallel $(2.01 \mathrm{~g})$ over the sponge (with a proper gap) than it does at an angle of $5^{\circ}(1.81 \mathrm{~g})$. At $0^{\circ}$, water is supplied and atomised evenly in nearly the whole contact area of the metal plate. However, when the plate touches the sponge at $5^{\circ}$, the thickness of the water layer formed between the metal plate and the sponge is not equal. Water is only atomised in a narrow area where the thickness of the water layer is suitable for atomisation. The amount of water supplied and atomised in this case is lesser than that at an angle of $0^{\circ}$.

\section{Collection distance between atomiser and collection paper}

This factor does not influence the atomisation process, only the mass of droplets collected.

With two different collection materials, collection paper and pad, the mass of droplets collected and the changes of cross-sectional area of droplet stream are, respectively, measured. The mass of droplets collected at $10 \mathrm{~mm}(2.0 \mathrm{~g})$ is a little more than that collected at $40 \mathrm{~mm}(1.83 \mathrm{~g})$ since most of the droplets have enough exiting velocity to reach $40 \mathrm{~mm}$ or more under the testing conditions.

The water print collected on the filter pad is an ellipse. In other words, the section of the stream of droplets is elliptical. As the contact area is rectangular, the water layer formed in between the plate and the sponge is in the shape of a cartouche owing to the surface tension of the water. The section of the stream generated is consequently close to an ellipse. Measured at 10 and $40 \mathrm{~mm}$ from the atomiser, the cross-section areas of the droplets stream are approximate ellipses of $17 \mathrm{~mm}$ (long axis) $\times 9.5 \mathrm{~mm}$ (short axis) and $18 \mathrm{~mm}$ (long) $\times 10.5 \mathrm{~mm}$ (short) respectively. It shows that the cross-sectional area of the beam expands only a little with the increment of the distance within the testing range.

On the basis of the above analyses, the value at which a better atomisation process presents for each factor is selected as the optimal value. The optimal operating condition is then obtained as shown in Table 3. 


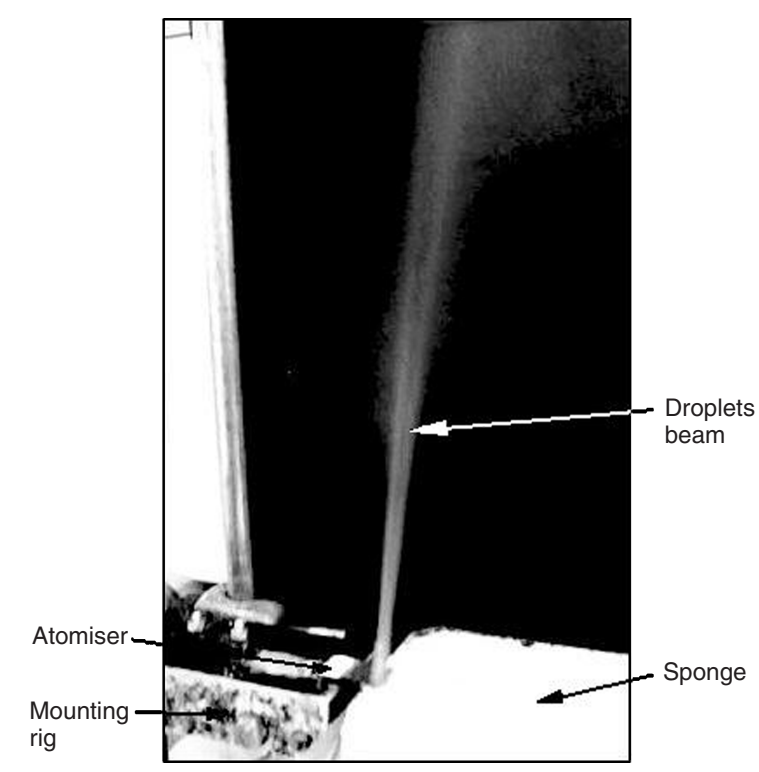

Figure 8 Snapshot of atomisation.

\section{VERIFICATION TEST}

A verification test is conducted with the optimal condition obtained in the previous section (Table 3 ). The same instruments and materials are used for the testing. The test is repeated three times. The atomisation time is set as $10 \mathrm{~s}$. Droplets are collected at $10 \mathrm{~mm}$ above the atomiser. A snapshot of the stream of droplets is shown in Figure 8.

The atomiser is placed horizontally on top of the sponge. The droplets are generated and sprayed upwards, forming a stream of droplets. It clearly shows that the spraying track of the droplets has strong directivity. The average traveling distance of the droplets, that is, the height of the stream, is measured as $0.4 \mathrm{~m}$. The average mass of droplets collected in $10 \mathrm{~s}$ is weighed as $3.3 \mathrm{~g}$ (or $330 \mu \mathrm{L} / \mathrm{s}$ ), which is more than the best record of $2.92 \mathrm{~g}$ in the trials discussed in the previous section. It demonstrates that the proposed optimal condition is the best one for the given atomisation process.

\section{FEASIBILITY OF THE ATOMIZER FOR THE APPLICATION OF OPHTHALMIC DRUG DELIVERY}

The feasibility analysis on the atomiser for ophthalmic drug delivery application is made against the conventional eyedroppers in the following seven aspects.

\section{Volume of the droplets collected}

As mentioned in the previous section, the droplets collection rate of the atomiser is $330 \mu \mathrm{L} / \mathrm{s}$ at $10 \mathrm{~mm}$ from the atomiser. To apply a desired volume of 5-15 $\mu \mathrm{L}$, time required for delivering this dosage would be only $0.02-0.05 \mathrm{~s}$. The high efficiency of an atomised drug delivery system brings the possibility of reducing the atomisation rate while still meeting practical requirements. It is impossible for a conventional eyedropper to supply such a small volume of drop precisely.

A microprocessor can be included in the driving circuit in a future study to control the atomisation process, by timing or voltage, so as to precisely control the dosage. This microprocessor can be configured to meet various drug delivery demands for individual drug solutions.

Meanwhile, the density of the minute holes in the metal plate can be reduced to lower the droplet generation rate so as to increase the drug-administering time if required. Since only one type of metal plate design is available currently, further study on this aspect will be providing more insight with new metal plate designs.

\section{Impact to eyes}

To the author's knowledge, there is no research data reported on the desirable droplets size of ocular drug for eye therapy and impact to the eye. This study uses the momentum per unit area as an indicator to make a comparison of the impact between droplets and conventional eye drops. Since there is no suitable instrument available for immediately measuring the impact force, momentum (mass multiplying velocity) is used as an alternative to estimate the impact on the eye. The momentum represents the action of both mass and velocity to the eyes.

Owing to the unavailability of a particle-measuring facility, the velocity of droplets is calculated with kinematic equations. Assuming the spraying droplets are acted only by gravitational acceleration, the average traveling distance is $0.4 \mathrm{~m}$, so the initial velocity of the droplets is calculated as $1.98 \mathrm{~m} / \mathrm{s}$. The velocity of the droplets at $10 \mathrm{~mm}$ above the atomiser is then $1.93 \mathrm{~m} / \mathrm{s}$.

Droplet mass can be calculated from the droplet size. According to Kong et al. (2002), the droplet sizes are mainly determined by the diameter of the mini holes on the metal plate. In this case, the droplet sizes are estimated as about 14-22 $\mu \mathrm{m}$ in diameter as the diameter of the mini holes on the metal plate is about $16 \mu \mathrm{m}$. For a droplet with a diameter of $20 \mu \mathrm{m}$, the mass is about $4.18 \times 10^{-6} \mathrm{mg}$. At $10 \mathrm{~mm}$ above the atomiser, the elliptical area of the stream is $217 \mathrm{~mm}^{2}$; there are about 2,650 droplets with a total mass of $0.011 \mathrm{mg}$ reaching the eyeball surface at the same time. Thus, the momentum per unit area of the droplets at $10 \mathrm{~mm}$ from the atomiser is

$$
0.011 \times 1.93 / 217=9.78 \times 10^{-5}(\mathrm{mg} \mathrm{m} / \mathrm{sec}) / \mathrm{mm}^{2}
$$

For a liquid drop from a traditional eyedropper, the mass is about $50 \mathrm{mg}$. When the eye drop is supplied vertically from $10 \mathrm{~mm}$ above an eye, its velocity, when reaching the eye, is calculated as $0.44 \mathrm{~m} / \mathrm{s}$. The contact area of the drop with an eyeball can be estimated as that of the drop's biggest section, that is, $16.4 \mathrm{~mm}^{2}$. The momentum per unit area is

$$
50 \times 0.44 / 16.4=1.34(\mathrm{mg} \mathrm{m} / \mathrm{sec}) / \mathrm{mm}^{2}
$$

It is obvious that the impact of the atomised droplets on the eyes is substantially gentler than that of a traditional drop, only $0.0073 \%$. It implies that impact to the eye is 
reduced significantly on using the new device compared to the eyedropper.

\section{Operation distance and orientation}

For a traditional eyedropper, the operation distance away from human eye is about $10-20 \mathrm{~mm}$ or even closer. The experiment result shows that the atomiser can perform very well in this distance range. When the atomiser is operated horizontally, the patient just needs to face down to apply the drug solution.

For future work, if a desired material that can absorb and hold the drug solution evenly is used to replace the sponge, the atomiser can be operated vertically and the patient just faces to the front. Assuming the distance between the eyes and the delivery device is $10 \mathrm{~mm}$, time required for traveling this distance is calculated as $0.005 \mathrm{~s}$ with an initial velocity of $1.98 \mathrm{~m} / \mathrm{s}$. The vertical drop of droplets in time is only $0.12 \mathrm{~mm}$ due to gravity, which is negligible on the drug delivery directivity. This means that the device would be able to operate vertically and consequently any angle between horizontal $\left(0^{\circ}\right)$ and vertical $\left(90^{\circ}\right)$ position. It would greatly improve the ergonomics of the use of the device.

\section{Distribution area of delivered droplets fitting eyeballs}

The elliptical section of the droplet stream is $17 \mathrm{~mm}$ (long axis) $\times 9.5 \mathrm{~mm}$ (short axis) and will fit the open eye of human being. According to Fledelium and Christensen (1996), the average length of the eye of a 3-year-old child is $22 \mathrm{~mm}$ and at age 8 to 13 and above is about $23 \mathrm{~mm}$. The width of a well-opened eye of an adult is about 12$15 \mathrm{~mm}$. The droplets applied can cover most of the eyeball area. This ensures even contact of drug with eyeball surface, which increases treatment effectiveness and efficiency.

\section{Power required for the device}

The optimal driving voltage $24 \mathrm{Vp}$-p can excite a very strong atomisation for drug delivery application with a rate of $0.33 \mathrm{~mL} / \mathrm{s}$. The required power is $2-3 \mathrm{~W}$. Each drug dose of $5-15 \mu \mathrm{L}$ can be supplied in only $0.02-0.05 \mathrm{~s}$, which demands very low energy consumption.

\section{PROPOSED PIEZOELECTRIC DRUG DEVICE}

A proposal of the ophthalmic drug delivery system is schematized in Figure 9. The device has four components: DC power supply, driving and control unit, drug supply unit, and atomisation unit.

The system is driven by DC power (batteries). The driving and control unit converts the DC power into an ultrasonic wave signal. The output voltage and operation time can be controlled using a microprocessor to generate a precise amount of drug droplets to suit the different drug dosage requirements of various drug solutions. When used, the delivery of drugs is simply a matter of switching

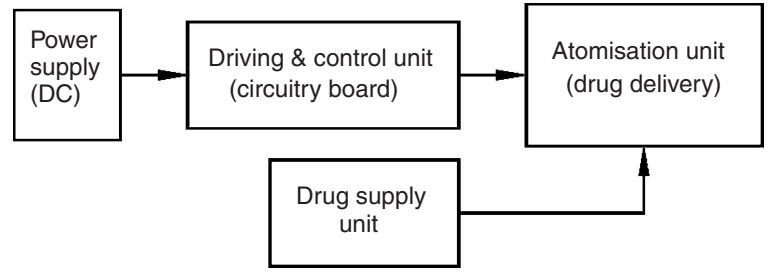

Figure 9 Structure of the ophthalmic drug delivery system.

on the power. The driving and control system will excite the atomiser to operate for a preset time; meanwhile the drug solution will be transferred to the atomiser through the supplying system and then the required dosage of drug droplets will be generated by the atomisation system and delivered to an eye.

The structure of the device is simple and can be fabricated in pocket size.

\section{CONCLUSION}

This research demonstrates that the performance of the atomiser is determined greatly by resonant frequency, driving voltage, atomiser's fixing method, and operating orientation. Besides, contact angle and gap between the atomiser and sponge, and collection distance influence the atomisation process to some extent but not as distinctively as the above four. An optimal driving condition is identified as follows: resonant frequency of $171 \mathrm{kHz}$, voltage $24 \mathrm{Vp}-\mathrm{p}$, atomiser operating horizontally, sticking method for fixing the atomiser, $0.5 \mathrm{~mm}$ gap, $0^{\circ}$ contact between the metal plate and the sponge, and droplets collection distance of $10 \mathrm{~mm}$. The atomisation rate under the optimal condition is $0.33 \mathrm{~mL} / \mathrm{s}$.

A feasibility analysis is also made against the traditional eyedropper. The analysis shows that the proposed atomising ophthalmic drug delivery device has significant advantages over the eyedropper:

- Delivery volume is precisely controllable;

- It provides a gentler spraying of drug to the eyeballs;

- Drug solution is evenly distributed across most of the eyeball area;

- It is easy to use;

- It is small and pocket-sized.

\section{REFERENCES}

Davies NM. 2000. Biopharmaceutical considerations in topical ocular drug delivery. Clin Exp Pharm Phys, 27:558-62.

Davison JR, Barnes P, Brace G. 2000. Dispensing apparatus. U.S. Patent No. 6,062,212. Washington, D.C.: U.S. Patent and Trademark Office.

Diamond J. 1997. Systemic adverse effects of topical ophthalmic agents: implications for older patients. Drugs Aging 11 352-60.

Diggory P, Franks W. 1996. Medical treatment of glaucoma - a reappraisal of the risks. Br J Ophthalmol 80 85-9. 
Fiscella R. 1998. Costs of glaucoma medications. Am $\mathcal{F}$ Health Syst Pharm, 55:272-5.

Fledelius HC, Christensen AC. 1996. Reappraisal of the human ocular growth curve in fetal life, infancy, and early childhood. Br 7 Ophthalmol, 80:918-21.

German EJ, Hurst MA, Wood D. 1997. Eye drop container delivery: A source of response variation? Ophthalmic Physiol Opt, 17:196-204.

Geroski DH, Edelhauser HF. 2000. Drug delivery for posterior segment eye disease. Invest Ophthalmol Vis Sci, 41 961-4.

Himmelstaein KJ, Baustian CL. 1997. Reversible gel-forming composition for sustained delivery of bio-affecting substances, and method of use. U.S. Patent No. 5,252,318. Washington, D.C.: U.S. Patent and Trademark Office.

Jarvinen K, Jarvinen T, Urtti A. 1995. Ocular absorption following topical delivery. Adv Drug Del Rev, 16:3-19.

Kong Y, Xu Y, Tay EH. 2002. Pulmonary drug delivery using droplets generated by Rayleigh instability-driven breakup of filaments. Proc SPIE Int Soc Optical Eng, 4937:144-73.

Kurosawa M, Watanabe T, Futami A, Higuchi T. 1995. Surface acoustic wave atomizer. Sensors Actuators, 50:69-74.

Lederer C, Harold R. 1986. Drop size of commercial glaucoma medications. Am 7 Ophthalmol, 101:691-94.

Lee YC, Millard J, Negvesky GJ, Butrus SI, Yalkowsky SH. 1999. Formulation and in vivo evaluation of ocular insert containing phenylephrine and tropicamide. Int $\mathcal{F}$ Pharm, 182:121-6.

Manton SM. 1989. Engineering for quality. In Bendell A, Disney J, Pridmore WA, eds. Taguchi Methods: Applications in World Industry. Vedford: IFS Publications, p. 13-36.

Maurice DM, Mishima S. 1984. Ocular pharmacokinetics. In Sears ML, ed. Handbook of Experimental Pharmacology, 69. Berlin-Heidelberg: Springer Verlag, p. 16-119.
Mishima S, Gasset A, Klyce S, Baum J. 1966. Determination of tear volume and tear flow. Inv Ophthalmol, 5:264-76.

O'Donoghue E. 1995. Beta blokers and the elderly with glaucoma: Are we adding insult to injury? Br 7 Ophthalmol, 79:794-6.

O'Sullivan E, Malhotra R, Migdal C. 2001. Prescription of eye drops. Postgrad Med F, 77:654-5.

Ross PJ 1988. Taguchi Techniques for Quality Engineering: Loss Function, Orthogonal Experiments, Parameter and Tolerance Design. New York: McGraw-Hill.

Saettone MF. 2002. Progress and problems in ophthalmic drug delivery. Business Brief Pharm, 167-71.

Shell J. 1982. Pharmacokinetics of topically applied ophthalmic drugs. Surv Ophthalmol, 26:207-18.

Shell J. 1984. Ophthalmic drug delivery systems. Surv Ophthalmol, 29:1779-82.

Sklubalova Z, Zatloukal Z. 2005. Systematic study of factors affecting eye drop size and dosing variability. Pharmazie, 60:917-21

Toda K, Ishll J. 1995. Operation performance of self-oscillation ultrasonic vibrating device for liquid atomization. $\mathcal{F a p ~} \mathcal{F} \mathrm{Appl}$ Phys, 34:5332-4.

Urtti A, Salminen L. 1993. Minimizing systemic absorption of topically administered ophthalmic drugs. Surv Ophthalmol, 37:435-56.

van Santvliet L. 1999. Optimisation of Eye-Drop Administration. Antwerp, $\mathrm{PhD}$ thesis.

van Santvliet L, Ludwig A. 2004. Determinants of eye drop size. Surv Ophthalmol, 49:197-213.

Winfield A, Jessiman D, Wiliams A, Esakowitz L. 1990. A study of the causes of non-compliance by patients prescribed eyedrops. Br 7 Ophthalmol, 74:177-80.

Yaniv E, Hadar T. 1994. Nasal irritation from eye drops. Am $\mathcal{F}$ Rhin, 8:153-5. 

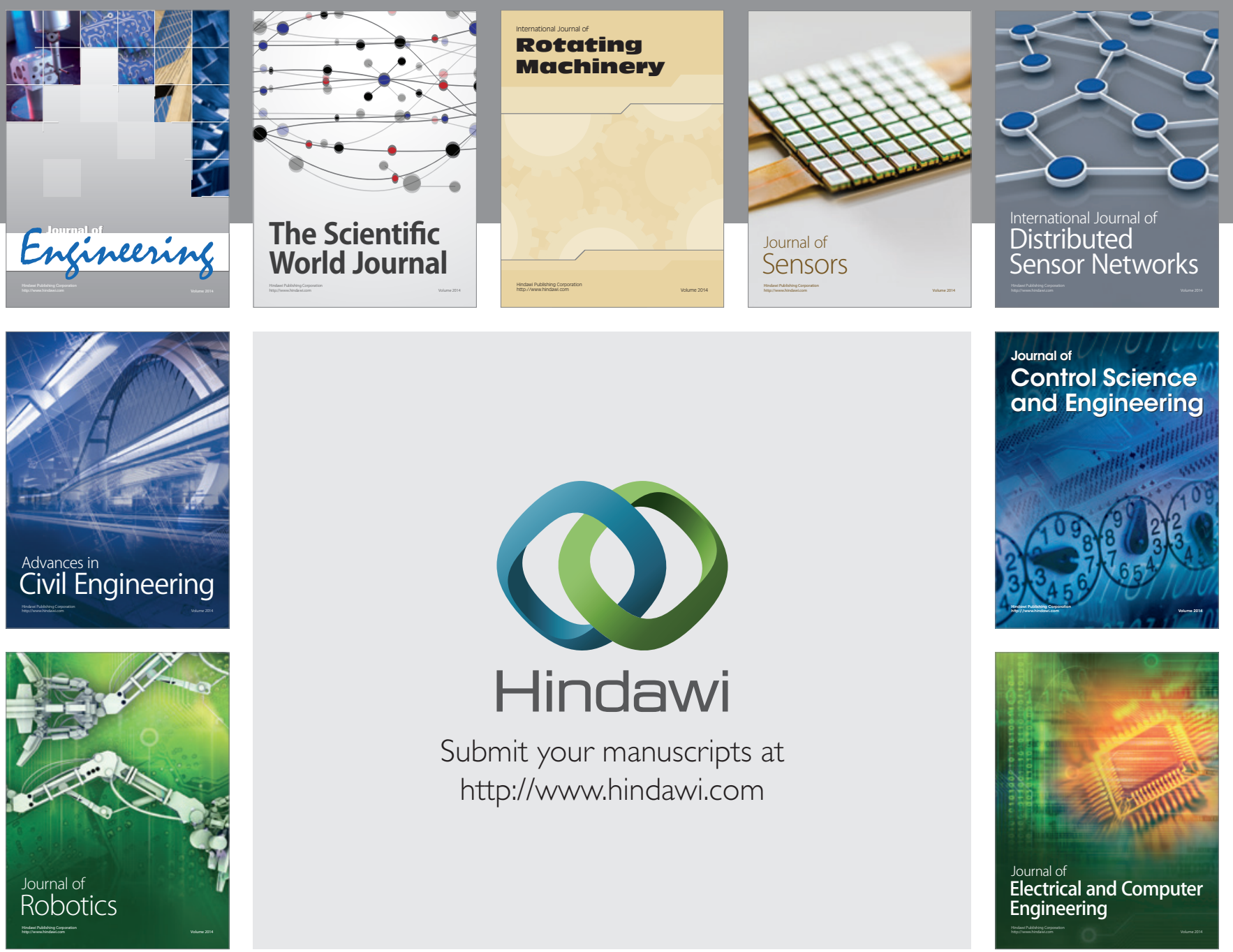

Submit your manuscripts at

http://www.hindawi.com
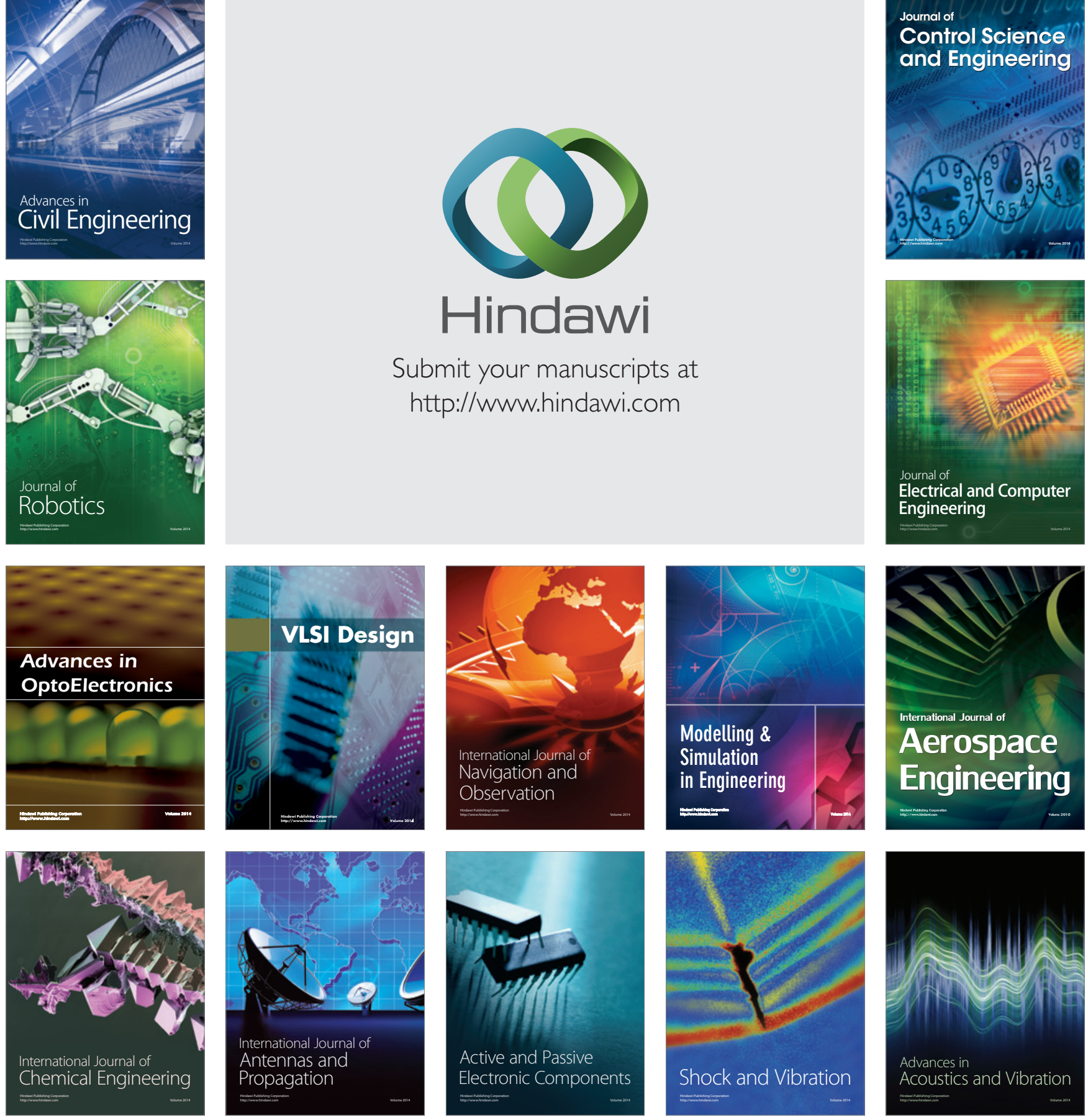